\title{
An Investigation into the use of Field Methods in the Design and Evaluation of Interactive Systems
}

\author{
Kelly Monahan \\ University of Sunderland \\ Sunderland, UK \\ (kelly.monahan \\ @ sunderland.ac.uk)
}

\author{
Mia Lahteenmaki \\ Nokia Research Center \\ Helsinki, Finland \\ (mia.lahteenmaki \\ @nokia.com)
}

\author{
Sharon McDonald \\ University of Sunderland \\ Sunderland, UK \\ (sharon.mcdonald \\ @ sunderland.ac.uk)
}

\author{
Gilbert Cockton \\ University of Sunderland \\ Sunderland, UK \\ (gilbert.cockton \\ @ sunderland.ac.uk)
}

\begin{abstract}
This paper reports the results of an international web-based survey on the use of field studies in the design and evaluation of interactive systems, which was conducted between December 2006 and February 2007. The results suggest that the advantages and disadvantages of field methods are generally well understood, but guidance is needed in their application and use. Field studies were most frequently used for understanding context, and respondents preferred a more varied approach to method use rather than following a defined methodology such as Contextual Design. Observations were rated as the most effective technique overall, although interviews appeared to be more frequently used. Significant areas of further improvement for field methods were identified as improvements in data collection/analysis tools and improvements in adaptability of methods.
\end{abstract}

\section{Categories and Subject Descriptors}

\subsection{H.5.2 User Interfaces}

\section{General Terms}

Human Factors

\section{Keywords}

Field ethods, User Centered Design, Survey, Ethnography.

\section{INTRODUCTION}

With mobile computing and digitalization expanding to various areas of our lives, we can reasonably expect the role of field studies in user requirements collection and usability evaluation to increase. Advances in technology demand more flexible and context sensitive methods to understand users and to appreciate how systems can be designed to support their tasks. However Dye [4] commented that "We do not have very good tools for understanding problem solving work, or for turning an understanding of problem solving work into design". He calls for a greater understanding of how to use ethnographic methods and field observations to design software.

Ethnography originates from anthropology and sociology, and has been used in these academic disciplines for at least several decades, but it was not until the 1990s that it began to be used in the field of HCI. At that time, researchers such as Hughes et al. [8] reported experiences of using ethnography in several projects and presented ways of using ethnographic field studies in system design. The method was later adapted for use as a

(C) The Author 2008.

Published by the British Computer Society technique for uncovering the real world character of work (in the context of CSCW system design). Although ethnographic field studies were fashionable during that period they were still a relatively untried method in system design, and their efficiency and usefulness still remained to be proven. HCI research has risen to this challenge, and in recent years field studies have been increasingly reported in the literature. However they are still not as widely utilized as some other User Centred Design (UCD) methods [13, 17] which is surprising as there is evidence to show that they have many benefits.

One example of the benefits of field studies is presented by Dray et al [3] in their summary of the field trials of the Microsoft Tablet PC. Having found that traditional UCD methods were insufficient to answer some of the crucial questions about usability, integration and adoption, they faced a difficult situation. Whatever methodology they chose had to be adaptable, allowing for changes in focus and direction, in order to allow them to study any emerging trends or address any gaps in knowledge that arose. It also had to be longitudinal and allow natural variations in usage to evolve. The "ethnographic field trials" they conducted integrated ethnography and usability testing, and included some preplanned structured evaluation activities to study key design features, and also some nonstructured interaction to study usage patterns and context. They found that their longitudinal study of this product allowed them to observe how the integration and usage of the technology evolved over time, created rapport with participants and also produced a greater opportunity for "opportunistic findings" that just happened to occur when they were present. The study resulted in the identification of a wide range of issues, including infrastructure and training issues as well as design changes.

McDonald et al [10] also reported on the benefits of field studies. They used an adaptation of Rapid Contextual Design [9] to conduct a field evaluation of an e-learning system, and their methodology included the addition of problem extraction and impact rating activities between the affinity building and design generation stages of the existing Rapid Contextual Design method. They theorized that these additional steps would improve problem understanding and ultimately lead to potential solutions that were better grounded in the data. Their findings indicated that formalizing the process of problem extraction assisted in capturing usability problems in a more structured way and related them explicitly to field data. Similar to Dray et al [3], McDonald et al discovered that they were able to identify a wide range of problems using field methods: $2 / 3$ of the problems they identified were related to the context of use rather than to the system being studied. They commented that it would not have been possible to identify such a broad range of issues using laboratory testing. On the basis of this they suggested that the iterative potential of field methods is extremely broad, encompassing not only design issues but also issues relating to product value and context of use.

We believe that field studies are an important part of system design and evaluation, but the available literature on field 
studies does not document the same kinds of simple procedures and "standards" possessed by literature relating to usability tests in laboratories [11]. As a consequence, people trained within different disciplines may perceive the same phenomena during field research but may view them in very different ways [5] Ethnography does not involve strict processes and preformulated study designs, so it is sometimes incorrectly considered to be an unsystematic method, a type of study that can be conducted without training or a deeper understanding of the methods involved [5]. This is a misconception that underestimates the expertise required in order to conduct field studies.

Our survey aimed to create a picture of current real-world usage of field studies, and represents important background research in this respect. No broad summary of current field study usage is currently available, so providing such a summary is of value to a wide range of researchers and practitioners. We hoped that this summary might provide some insight into why field studies are not as widely used as one might expect, and as far as we are aware these issues have not been investigated in any existing survey study to date. The decision to gather this data using a survey was made because we felt that it was important to gain as wide a view as possible of the current state of field research, and the only way to reach a diverse sample of academics and practitioners worldwide was via a web-based survey. This paper is intended to provide an overview of the survey data we gathered and offer a snapshot of current field study usage, and we feel that the broad focus of the paper is one of its main assets. We believe the paper is of significant value in identifying gaps in current knowledge and providing directions for further research, and we anticipate that the results will support awareness of where new value could be delivered through field method improvement.

\subsection{Previous Surveys}

Several surveys have been conducted on UCD practice, especially in the first half of the current decade (e.g. [6], [7], [12], [13], [14], [15]). Most of the studies aimed to rank the methods and their experienced effectiveness in order to provide an overview of the general status and practice of UCD work. Some surveys also included organizational issues and information about the status of usability expertise in organizations ([6], [13]). However their focus on usability work or UCD at the general level means that our current knowledge regarding the use of specific tools or methods with field studies is quite limited.

A survey on UCD practice by Vredenburg et al. [13] reports the state of the art in UCD based on 103 responses from the US and Europe during 2000-2001. The survey was conducted among ACM CHI conference attendees and UPA members. It concentrated on the state of UCD in organizations and how effective it was considered to be. The most important finding regarding field studies emerged when ranking UCD methods according to their importance. When asked to rank the five most important UCD methods on the basis of their actual impact on product development (including user satisfaction, results in the market, and cost savings) field studies (including contextual inquiry) were considered to be the most important method, together with user requirements analysis. Yet field studies were used less than more inexpensive methods which were considered to have less impact. According to the research, respondents seemed to be particularly cost-conscious in their evaluation of UCD methods, so this may have been one reason for the reported lack of utilization of field studies.

An earlier survey on strategic usability was conducted by Rosenbaum et al. [12]. Strategic usability was defined as "embedded usability engineering in the organizational processes, culture, and product roadmaps". The survey described the methods considered to increase the strategic impact of usability research within companies, and it found that field studies are very effective in contributing to strategic usability. Field studies (other than contextual inquiry) were rated as 6th among 29 different methods studied; contextual inquiry was rated as 12th. However, similar to the aforementioned UCD survey [12] this survey also found that while many field methods were rated as highly effective they still had relatively low usage.

The most recent survey on UCD was conducted by Venturi et al. [15] and concentrated on organizational adoption of UCD and its integration into business processes. They noticed that according to their survey, UCD activities are typically carried out during the early phases of the product life cycle: during requirements, analysis and design. The survey revealed that most of the participants conducted qualitative evaluations as opposed to quantitative evaluations, but it did not produce results on field methods separately.

\subsection{Our Approach}

The surveys mentioned constitute more or less the entire body of previous survey work relating (however distantly) to field study usage, and it is clear that there is little knowledge to build upon. For this reason we decided to take a very broad focus in our survey, and we hope to provide a basic foundation of knowledge upon which further investigation can be founded.

The aim of our survey was to investigate the field methods used by practitioners and to learn about the perceived usage and effectiveness of the methods, the utilization of results, and the challenges faced when using these methods. We were also interested in the perceived advantages and disadvantages of both lab and field methods, and in the factors influencing a practitioner's choice of methods. In addition we wanted to explore possible improvements to field methods that would make them more useful to practitioners.

Our approach differed from previous surveys in that we focused specifically on field studies and related issues. According to the existing literature it seems that field studies are considered to be an important and effective method, but they are under-utilized and there is not much measured information about the current status of field study use. This study will go some way towards rectifying that situation, and towards identifying the issues that may be inhibiting wider utilization of field methods.

The results of the survey will provide an updated view on current practices in industry and academia, and knowledge of the current state of matters is the first step towards improvement. Understanding the state of the art is a necessary condition for forward movement. The findings presented here represent a recent snapshot of field method utilization that we hope can be used to inspire areas for further research in this burgeoning field.

\section{THE SURVEY}

The survey was conducted as a web based questionnaire, and respondents were invited to complete the questionnaire by means of invitations published on professional mailing lists such as ACM SIGCHI and CHI/NordiCHI announcements lists. Invitations were also published on the UK UPA discussion forum and in Usability News. The target respondents were usability practitioners in education and industry, with varying levels of experience and knowledge in field studies. It is difficult to estimate the sample size, but based on the membership of the email lists we targeted and the high number 
of responses received we believe that we have reached our major target sectors.

The survey was entirely anonymous to prevent bias. We also felt that anonymity would allow respondents to report method usage and challenges experienced without concerns for their professional or corporate reputation.

The survey contained 21 questions on the following topics:

- Demographics (country, sector, size of employer, etc)

- Type of studies conducted (lab or field), knowledge and experience in field studies

- Methods used, effectiveness of methods

- Utilization of results

- Challenges faced when conducting field studies

- Factors influencing choice of methods

- Advantages and disadvantages of lab/field methods, possible improvements to field methods

These topics were chosen because they encapsulated the aim of the study, which was to find out who was doing what type of studies and why, for the benefit of whom, and what challenges they faced in doing this. Those respondents who indicated that they did not conduct field studies were given a subset of questions relating to demographics, knowledge of field studies, factors influencing choice of methods, advantages and disadvantages of lab/field studies, and possible improvements to field studies. They were not requested to complete the questions relating to usage of field studies, utilization of results and challenges faced, as these were oriented more toward field study practitioners.

Our aim was not to define what constitutes a field study, as there is already a prolonged debate on this subject, which is worthy of a paper in its own right. There is already a wealth of published material relating to definitions of field studies, and defining field studies was not an area that the survey or the paper was designed to address. For the purposes of this survey, a field method was defined as "a study in the user's own environment" and a lab method was defined as "a study in a researcher-controlled environment". Since different ways of working have to be divided and labeled in some way, this division seemed to be logical, and is widely employed in existing literature; we would expect the audience to have a broad understanding of these terms. Doing research in each environment has different pros and cons, and no suggestion is made that either approach is superior to the other, or that one can be used to replace the other; they are envisaged as complementary. Although our main interest was in field studies, if the survey related only to field studies it would have gathered respondents who are biased towards field studies, and in order to avoid bias we wished to obtain a broader cross-section of respondents who conduct all types of studies.

The survey as it stands does not contain any data which could hope to address the question of what constitutes a field study, therefore any definition we could provide would be based solely on previous literature and not on data, and for this reason we have chosen to adhere to our original broad definition and have not attempted to further define field studies.

Neither was our aim to investigate field study methods, as this is a very large subject in its own right, and there are already numerous studies of field methods [e.g. 16]. Our aim was to find out more about what happens in practice today and to look at how practitioners use field methods (assuming the broadest possible definition of "field methods") since this is the information which the current literature is lacking.
This survey was a combination of questions from three individual surveys (unpublished) that were previously conducted by the authors at HCI2005, HCI2006, and NordiCHI2006. The response rates for these questionnaires were acceptable but not particularly high, so the authors decided to combine the surveys and run them as an international web-based survey. The web-based survey was piloted twice in September and November 2006.

The survey was live between December 2006 and February 2007, and 317 responses were received in total; however due to the fact that not all respondents completed all questions the total figures for each question didn't always add up to 317.

\section{RESULTS}

\subsection{Respondents Profile}

The greatest numbers of responses were received from the US, the UK and Finland (in that order). Smaller numbers of responses were also received from various European and Asian countries, and from Australia, Canada, Mexico, Brazil and Israel. Of the 317 responses received, the majority of respondents $(172$, or $54 \%)$ conducted both field and lab studies. Seventy-five respondents (24\%) conducted only lab studies, and sixty-four respondents $(20 \%)$ conducted only field studies. Six respondents $(2 \%)$ did not conduct either lab or field studies. Of those respondents who conducted both lab and field studies, they tended to conduct fewer field studies than lab studies. The majority of them reported that the proportion of studies conducted in the field was " $10 \%$ or less" ( 50 respondents) or "Less than half" (50 respondents). Only eleven respondents conducted more than $90 \%$ field studies.

The majority of respondents (205) were from industry, a smaller proportion were academics (80), and a minority were research students (32). The numbers of academics and students doing only lab studies, only field studies, or both types of studies showed only a small variation, but a large majority of those from industry did both types of studies.

Of those respondents from industry, the majority belonged to various diverse sectors. The largest number of respondents in a single sector came from user research, with software and telecoms in second and third place (see figure 1).

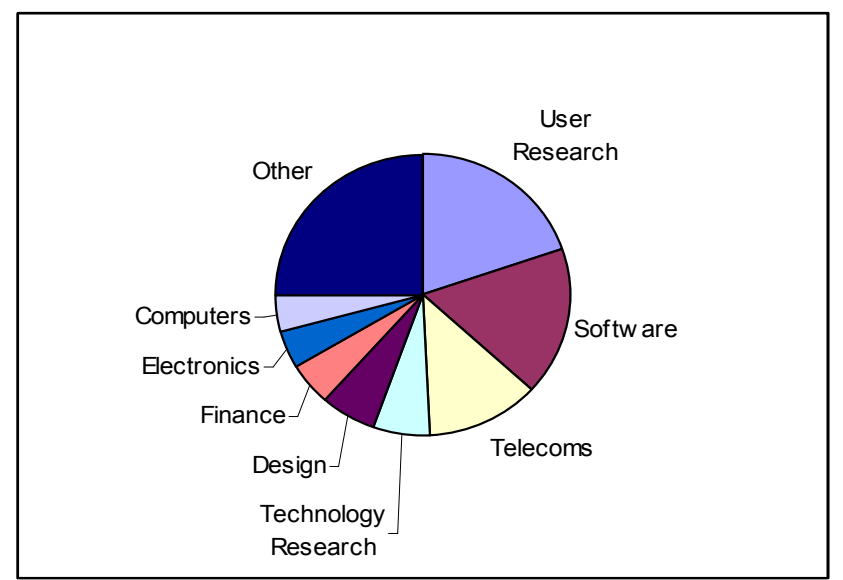

Figure 1: Industry respondents by sector

The majority (76\%) of respondents rated their experience and knowledge of field studies to be either intermediate or expert. The remainder $(24 \%)$ rated themselves as beginners or said they had no expertise. The majority of respondents who conducted only field studies rated themselves as expert, while those who did only lab studies rated themselves as intermediate or beginner. For those who did both lab and field studies the 
numbers were evenly split between intermediate and expert (see figure 2).

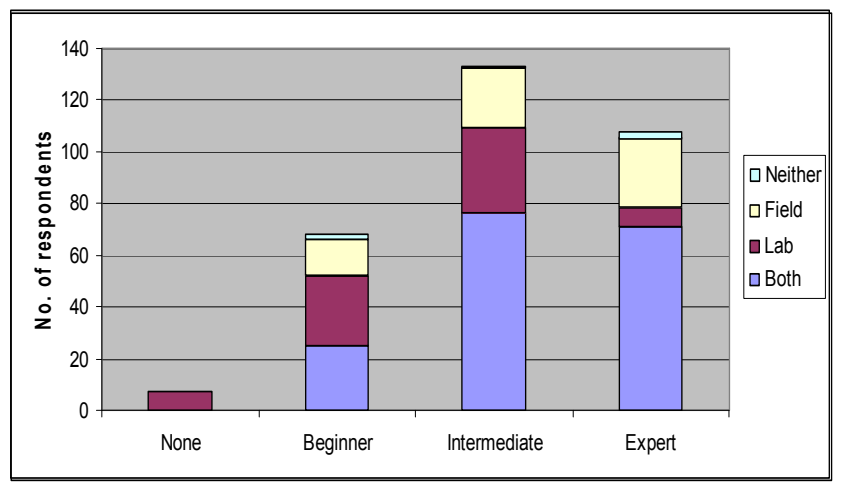

Figure 2: Knowledge of field studies split by type of studies

\subsection{Usage and effectiveness of methods}

\subsubsection{Usage of Methods}

A selection of methods commonly used in field research were listed, and for each method they had used the respondents were asked to indicate the purpose for which they had used it. It was possible for respondents to mark multiple uses for a single method. It is common practice in surveys such as this to provide respondents with a selection of possible responses, and the options we chose were derived from literature review and brainstorming. The options we eventually included in the survey were the most popular ones in our previous (unpublished) small-scale surveys.

Overall, understanding context was the purpose for which field studies were most frequently used. Observations, Contextual Design, diary studies and probes were all used primarily for understanding context, while interviews were used slightly more frequently for requirements gathering, and usability testing was used primarily for evaluation/validation (see figure 3 ). This focus on the use of field methods for gathering contextual information excludes much of their potential for use in other phases of development.

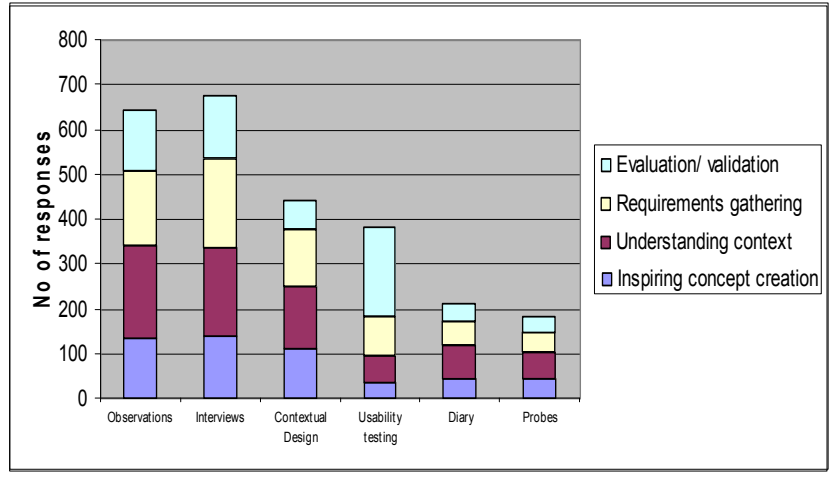

Figure 3: Usage of field methods

\subsubsection{Effectiveness of methods}

Having established which research methods were used and for what purpose, we also wanted to investigate why the researchers had chosen to use those methods in the first place. One aspect of this choice is likely to be the perceived effectiveness of the method. Respondents were given a small selection of methods and techniques and asked to estimate, according to their experience, the effectiveness of the methods for field evaluation purposes. When a Likert scale was applied to these responses, observations were rated as the most effective method overall, with Contextual Design in second place and interviews coming third. Usability testing, probes, and diary studies followed in that order of priority (see figure 4).

Looking back at figure 3 we can see that interviews were the most frequently reported method (receiving the highest number of ratings) however they are only the third best method in terms of effectiveness. The most effective method (observations) was the second most reported method in figure 3 . This inconsistency between method effectiveness and usage has been commented on previously by other researchers ([12], [14]).

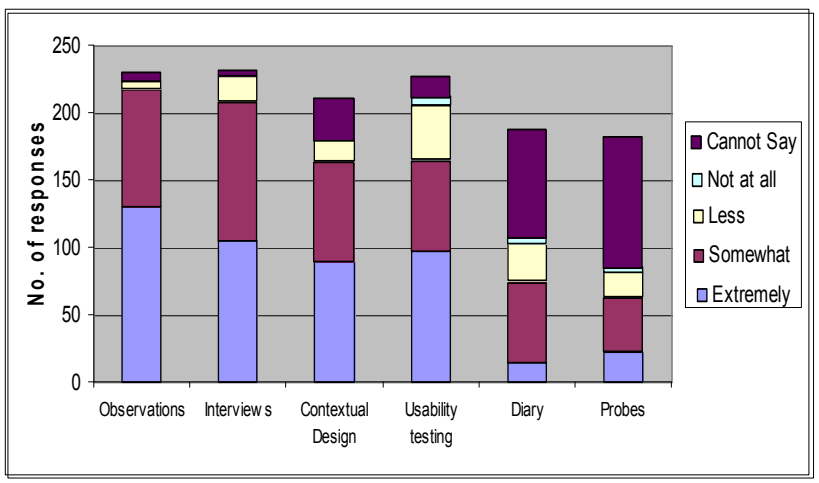

Figure 4: Effectiveness of methods

\subsection{Factors influencing choice of methods}

We also provided respondents with a small selection of possible factors that might influence choice of methods, and asked them to select all that applied. As for previous questions, the options we chose were derived from literature review and brainstorming, and from the results of our previous (unpublished) small-scale surveys. Research questions/aims of study and time constraints were the factors which most affected choice of methods overall (see figure 5). These results held true for those respondents who conducted only lab studies, but quality/relevance of results replaced time constraints as the second most important factor for those respondents who conducted only field studies. Since previous research (e.g. [12], [14]) has already established that there does not appear to be a direct relationship between method effectiveness and usage, these additional factors must be exerting a significant influence on choice of methods. A further analysis of the data showed that a higher number of academics and students selected research questions/aims of study as an influencing factor than selected time constraints, whereas the situation was reversed for those who worked in industry; this illustrates the differences between priorities and constraints in different sectors.

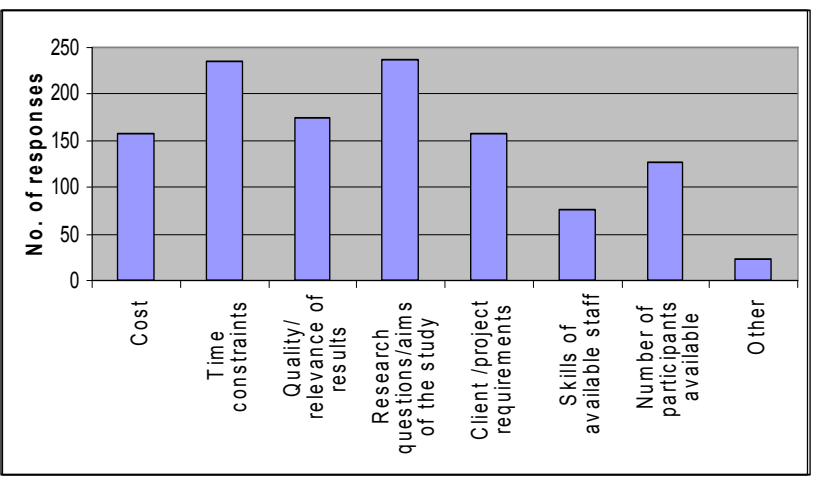

Figure 5: Factors influencing choice of methods 


\subsection{Aims of respondents' research}

Field studies are notorious for difficulties in defining focus, and heated discussions have taken place regarding how specific the focus should actually be. However we believe that whether the focus is purposely loose or strictly defined, the aims of the study can still be written down at some level to clarify the aims of the study to all involved. We were interested to discover how often studies had aims written in a formal plan, rather than just allowing the key points to emerge from the data. It was our assumption that the maturity of research practice can at some level be estimated based on whether or not the aims of the study are written in a plan. We expected that the results of this would be rather different for lab and field studies. However we discovered that although lab studies more frequently have planned aims, field studies are not too far behind (see figure 6). When a Likert scale was applied, "Often" emerged as the average response for both lab and field studies. Unfortunately we did not gather any data on the iterative nature of the aims for each type of study. We would expect that the aims of lab studies would be more likely to be fixed, while the aims of field studies would be iterated, perhaps in a manner similar to that described by Dray et al [3]. This increased iterative potential of field methods was also suggested by McDonald et al [10].

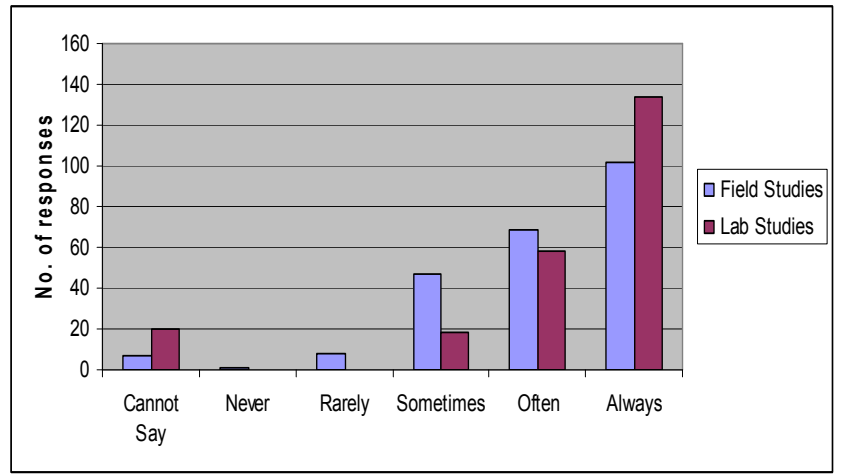

Figure 6: Frequency with which lab and field studies have aims written in a plan

\subsection{Utilization of Results}

\subsubsection{Utilization of results by organizational function}

The aim in finding out who uses the respondents' study results was to better understand what kind of research the respondents are doing and who they feel are the audience for their results. The question of who utilizes the research data is relevant information for practitioners who conduct field studies and indicates the current status and relevance of the research in general. While the existing literature contains descriptions of situations in which field studies have been used (e.g. [3], [10]) we found no statistical data about current utilization of field study results. Respondents who stated that they conducted field studies were asked the question "Who utilizes your research results?" The question was formulated so that respondents would select all alternatives that applied, in order to provide information about all utilizing group/organization functions not just the most typical ones.

The utilization of field research results by function is visible in figure 7. Overall, field research results had a wide spectrum of use among many groups and organizational functions. When looking at the results, Design and Development are clearly the most popular alternatives, each selected by over $70 \%$ of respondents. Research (48\%), Academic/HCI Community $(45 \%)$ and Marketing (45\%) were also utilizing the research results considerably. These figures seem quite high, but we must remember that they do not show the direct frequency of utilization by these functions since we felt that would be too difficult for respondents to estimate. Instead it shows the number of respondents whose results have sometimes been utilized by these functions. Communications is likely to be a major stakeholder for most web related projects, and its reasonably high popularity among respondents from industry might be partly due to that. The frequency of occurrence of Customer Support/After Sales Services (28\%) was also a surprise; the majority of those who selected this response were practitioners in industry, and probably at least part of this is again due to building and maintaining web interfaces for customers. There is also the possibility of results being passed to Customer Support/After Sales Services to support their response while development staff are introducing a fix. HCI specialists often work closely with customer support, both to benefit from support log data, and to proactively provide ideas and insights for front-line support, and this may be one possible explanation for the relatively high frequency with which this group utilizes research results.

Research results from academics were naturally mostly utilized by Academic/HCI Community and Research. However many academic respondents also indicated that they provided information for design or even for development. Some of this activity is likely to take place in companies and some in publicly funded projects, but still these figures concretize the fact that academia and industry tend to be quite well mixed, at least in the respondent set that was approached by our recruiting channels.

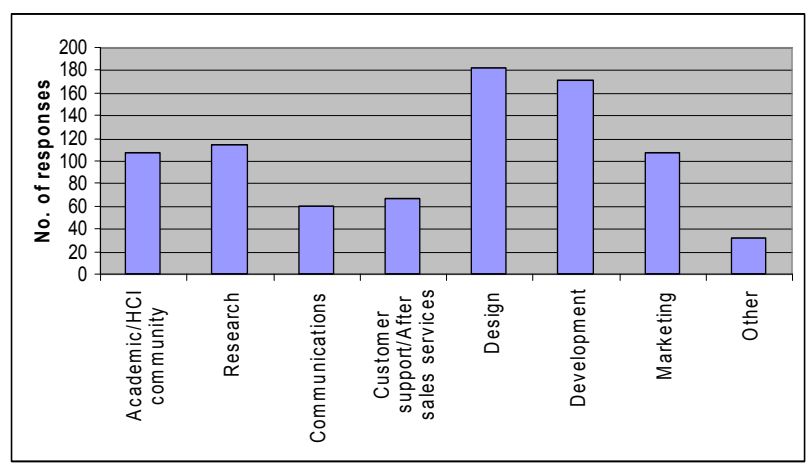

Figure 7: Utilization of field research results by function

\subsubsection{Efficiency of utilization}

The respondents who had stated that they conducted field studies were asked to rate how often they felt that the results of both lab and field studies were efficiently utilized. The results are shown in figure 8. According to the graph, lab study results appear to be efficiently utilized slightly more often than field studies. We know that 172 respondents conducted both field and lab studies and 64 respondents conducted only field studies, which explains the slightly higher number of "Cannot Say" responses for lab studies. However the number of "Cannot Say" responses for lab studies (11) does not correspond to the number of respondents who conducted only field studies (64), so we cannot know whether these respondents were able to base their response on personal experience as requested in the survey question. This means that some of the responses for lab studies may be subjective responses based on the respondent's knowledge and not grounded in practical experience. This is a flaw in the questionnaire design which was unfortunately not picked up during the piloting process. 
One problem in gathering this data is that sometimes usability practitioners lack information about how the results of their research are actually utilized, especially when working for an external company.

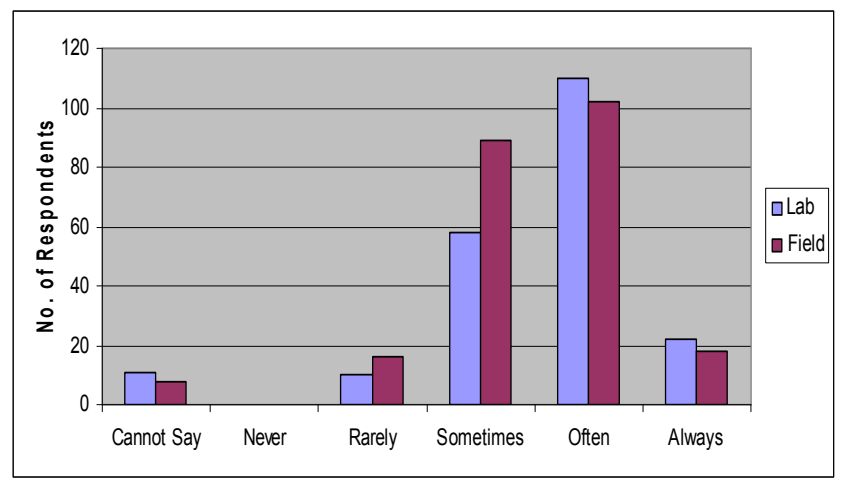

Figure 8: Utilization of research results by efficiency

On average, the utilization of results is optimal neither for field studies nor lab studies. When applying a Likert scale to the results (1-5, 5 being the highest), the average score for field studies was 3.54 and for lab studies 3.72. This means that the average response for both types of studies was that the results are "Often" efficiently utilized, although the results of lab studies appear to be slightly better utilized overall. This reveals the slightly alarming fact that in a relatively high proportion of cases the research results are not efficiently utilized. Considering effective utilization of results as a visible measure of success and as a measure of the impact of a study in an organization, having such a low number of responses in the "Always" category is unsatisfactory for both field and lab studies.

\subsubsection{Quality of results}

The lack of optimal utilization of research results may bear some relationship to the quality of the results produced. The survey included questions asking respondents to estimate the frequency with which field studies are pressured by cost constraints and deadlines, to the extent that there is an impact upon the quality of the results. The results of this are visible in figure 9. When a Likert scale was applied to each of these, "Sometimes" emerged as the average response for both cost constraints and deadlines. Despite this, it is still of concern that so many respondents (73 and 17 respectively) said that field studies are often or always pressured by deadlines which affect the quality of the results, and an even higher number of respondents (74 and 21 respectively) said that field studies are often or always pressured by cost constraints which affect the quality of the results.

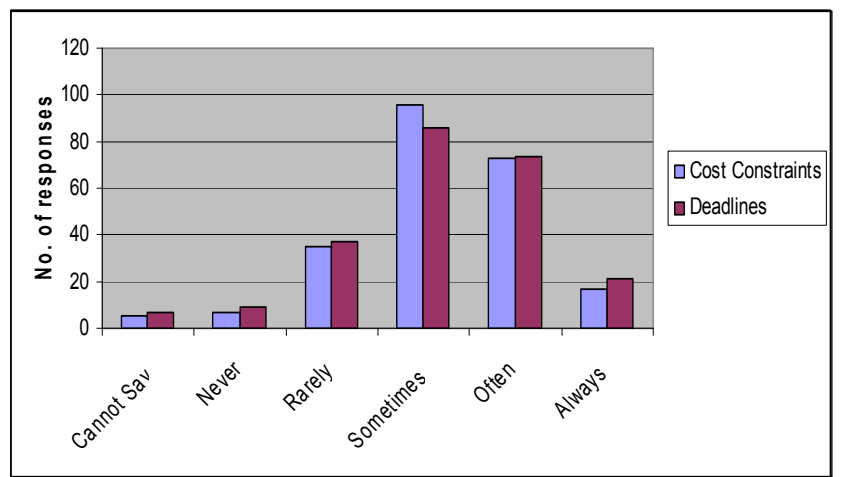

Figure 9: Frequency of field studies being pressured by cost constraints and deadlines

\subsection{Advantages and Disadvantages of Lab/Field Studies}

Respondents were given a set of multiple choice questions and asked to identify the main advantages and disadvantages of lab and field methods. The list of multiple choices was randomized per responder on the web questionnaire, so that the order of choices would not bias the final response set.

\subsubsection{Advantages of Field Methods}

By far the main advantage of field methods was their real world context, followed by the rich data set they generate and the fact that tasks emerge from the user (see figure 10). This order of advantages was the same for those respondents who conducted only field studies, but the latter two options were rated equally by those respondents who conducted both lab and field studies, and were reversed for those respondents who conducted only lab studies. Other advantages mentioned included improved ecological validity, usefulness of results from both technology and business perspectives, gathering unanticipated data that perhaps would not emerge in lab studies, and driving innovation.

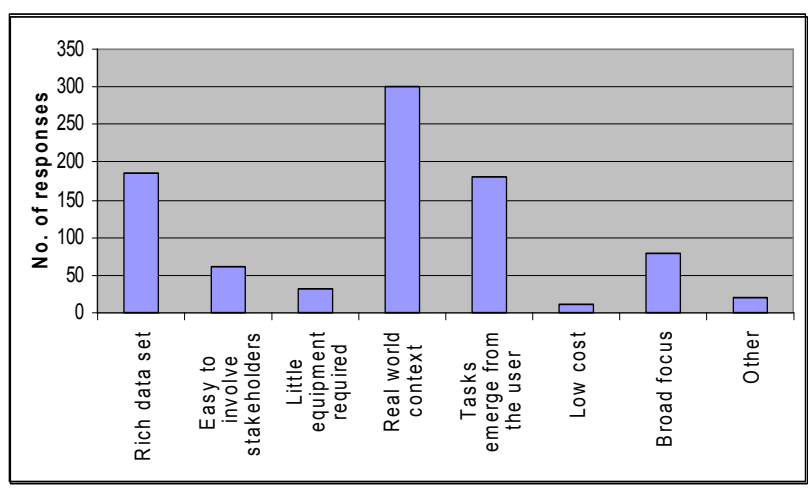

Figure 10: Advantages of Field Methods

\subsubsection{Disadvantages of Field Methods}

The main disadvantages of field methods were that they are potentially labour intensive, studies tend to have a long timescale, and there may be difficulties with data analysis (see figure 11). The order of the latter two options was reversed for those respondents who conducted only lab studies. Other disadvantages mentioned included difficulties with participant recruitment and availability, lack of repeatability, lack of buy-in or difficulty gaining approval for studies, large volume of data, and constraints imposed by project owners.

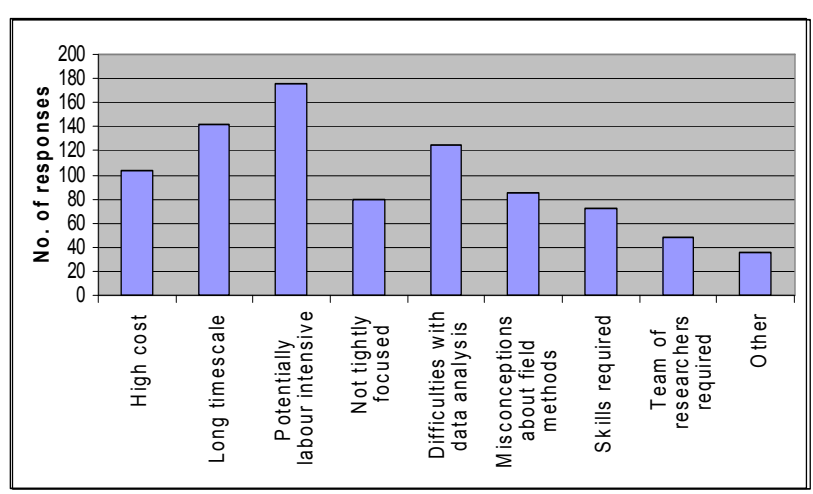

Figure 11: Disadvantages of Field Methods 


\subsubsection{Advantages of Lab Methods}

Overall, the main advantage of lab methods is that they are focused, closely followed by the fact that they give more control over the user's tasks (see figure 12). The results for those respondents who conducted both lab and field studies were the same as the overall results, but those respondents who conducted only lab studies or only field studies rated these two options equally. Other advantages mentioned included ease of stakeholder involvement, greater control over the environment, low costs, methods are more convenient and less labour intensive, there is a focus on interaction with the device rather than the use context, and it is easier to gain approval from management for lab studies.

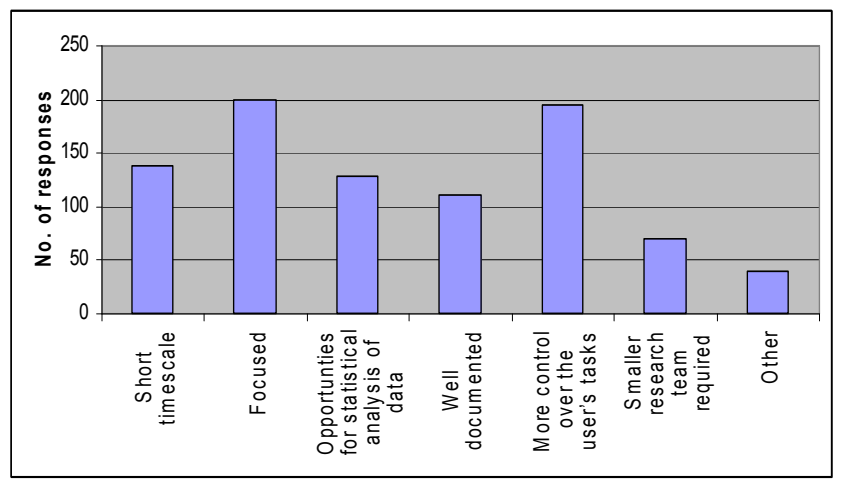

Figure 12: Advantages of Lab Methods

\subsubsection{Disadvantages of Lab Methods}

The main disadvantage of lab methods is that they do not look at tasks in context, followed by the fact that tasks emerge from the researchers, not from the users (see figure 13). These results were true for all groups of respondents. Other disadvantages mentioned included lack of ecological validity, difficulties with participant recruitment and availability, difficulties with task selection, tendency to focus on short term usage only, and inability to investigate what the system lacks.

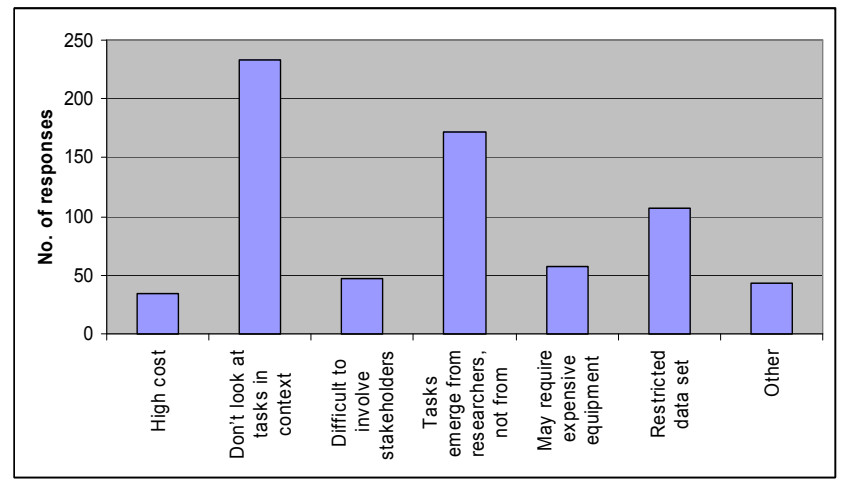

Figure 13: Disadvantages of Lab Methods

\subsection{Possible improvements to field methods}

Participants were asked to describe in an open question how field methods could be improved to be more useful to them. The data was analysed using a bottom-up card sort method, in which the responses were printed out and grouped with similar responses, and the resulting categories were labeled at the end of the process. If individual respondents had provided more than one distinct suggestion these were treated separately when grouping the responses. Altogether 69 suggestions were made for field method improvements; the results are summarised below.

\subsubsection{Improved methodologies}

Sixteen respondents felt that improvements in the methodologies used would be beneficial, particularly in the area of integrating field research with model-based design or traditional market research. One respondent commented that:

"A shortened version of contextual inquiry would be nice. Though Holtzblatt and Beyer wrote a book on this, it seems to be very similar to their full-blown model. I like the idea of doing contextual inquiry, but then stopping at affinity diagrams."

The affinity diagram stage of Contextual Design is the point at which McDonald et al [10] argued that the method required modification. They pointed out that generating design ideas via inspection of an affinity diagram "may not result in direct mappings between usability problems and design fixes". They advocated the use of Structured Report Formats (based on those used in [2]) to address this difficulty; these are simply sheets with a number of different headings on them describing the different aspects of a usability problem, which are designed to assist participants in writing usability problems in a structured way. McDonald et al [10] found that these report formats proved to be extremely successful at reporting problems in detail and grounding them in the data.

One aspect of methodologies that (four) respondents believed required considerable improvement was data analysis techniques. They highlighted the time-consuming nature of existing techniques, and one respondent called for "more explicit explanations of how to do analysis rather than intuitive processes of analysis". Even respondents who didn't advocate any particular methodology still indicated that it would be beneficial to have some alignment between field research goals, data collection methods, data analysis and problem extraction. Other issues raised by respondents included the need for standardization of methodologies, the requirement for strong guidelines and concrete examples for practitioners to follow, the development of less intensive methods, improved participant selection procedures, and techniques for accounting for cultural differences between participants.

\subsubsection{Improved Tools}

Fourteen respondents commented on the requirement for improved tools for data collection, analysis and communication of results, with a particular orientation toward making these tasks less time consuming. Suggestions included:

- Software for automatic transcription and translation

- More efficient methods for data entry and synchronizing multiple sets of data

- $\quad$ Equipment for remote viewing of user tests

- Small light devices without energy consumption problems

- Better digital recording methods for photos, voice and video

- Higher quality digitization and tagging of video

- Data collection tools to automatically sort data into groups

- Automated electronic resources and report generators

- Methods for easier matching between different types of recording materials

- An easier, more integrated way of recording events in the field without interrupting the flow or attracting attention 
Some of these points relate to the need for better methods of doing remote testing and minimizing intrusion during the data collection process. The other main theme of responses is the need for better software tools to automate data collection and analysis processes. These tasks are frequently time consuming, and this leads to reduced opportunity for data collection and analysis in a given time period. It could be argued that the development of such tools could relieve time and budget pressures to some extent, since speeding up data collection and analysis processes would allow researchers to either collect more data in a given time period, or to shorten the timescale of studies and reduce labour intensiveness, thereby reducing the budget required. As labour intensiveness, long timescales and difficulties with data analysis were revealed by the survey to be the three main disadvantages of field methods, this is an area in which significant improvements to methods could be rapidly achieved.

\subsubsection{Adaptable methods}

Seven respondents indicated that adaptability of methods is important, as they tend to adapt methods to suit the requirements of each individual case. Two of these respondents commented that each method has its advantages and disadvantages, and a multi-method approach allows the collection of varied data - the method chosen must reflect the requirements and match the research phase, and also be appropriate to the project goals and constraints. This multimethod approach was also employed by Dray et al [3] although they noted that combining methods produced highly heterogeneous data that could be difficult to manage and interpret, and pinpointing the best combination of tools was complex and required pilot tests in advance of the actual study. However once the optimum combination of tools/methods is identified it may be possible to reuse it in subsequent studies; one survey respondent informed us that "The main change that makes these (adapted) methods more useful is to use them across repeated engagements with the same client, so they become more integrated with their product lifecycle."

\subsubsection{Improved awareness and understanding of the value of field studies}

A total of four respondents called for a wider comprehension of field methods, both among researchers and participants - one of these respondents suggested seminars by high profile speakers as a possible method of raising awareness. Another respondent felt that this lack of understanding of field methods can lead to the belief that spending time with customers is not real work and is not of value. Lack of understanding on the part of the participants was also reported as problematic, as this can lead to misconceptions about what the field study will actually involve, and participants can be "a little bit weirded out when we start following them around everywhere". Rosenbaum et al [12] discovered that lack of understanding about usability was a major obstacle to strategic usability in organizations.

Improved awareness of field studies could perhaps be achieved through better reporting - both reporting of results to key stakeholders and reporting of studies to the HCI community (as proposed by two respondents). Rosenbaum et al [12] also identified a need for better ways to communicate the impact of results as being one of the key obstacles to strategic usability.

\subsubsection{Buy-in and integration between key people}

Integration between key people such as designers/developers, usability practitioners, project managers and participants is particularly important in field studies, and was commented on by a total of ten respondents. Two of these respondents indicated that it is important that this integration should happen from the earliest stages of the project, and that all parties should accept this integration.

Unfortunately this is not always the case, and four respondents commented that achieving buy-in was a problem, and that management buy-in was particularly important in order that visits were not seen as an intrusion. Uncooperative managers and lack of management buy-in are a common problem when conducting field research, and one respondent suggested that there should be a greater reliance on more experienced business people whose focus is on the project rather than on more personal objectives. This problem can be exacerbated when managers are the gatekeepers of the research; one respondent claimed that he would much prefer to have direct access to participants.

Even integration between usability researchers themselves can be problematic. There was some disagreement among respondents as to whether group work was a benefit or a hindrance to field research. One respondent suggested that methods should enable group work, as group work can help with "capturing issues, recognizing biases, and speeding up the distillation process". In contrast, another participant felt that giving one person sole responsibility for planning and executing the study was the best approach. As the necessity of training and practice in the use of field methods was commented on by two additional participants, perhaps group work is beneficial in the sense that it allows group members to share skills and assist each other? Certainly in Contextual Design [9] group work is an important part of the methodology, as it helps to reduce bias.

\subsubsection{Initial data collection}

Two respondents highlighted the importance of initial exploratory visits to the field in order to give some prior understanding of the participants and the environment, thereby allowing the researcher to maximize the observation and interview time available. This is essentially a method of gaining focus, which can be a particular difficulty with field studies as the methods are often required to be flexible and responsive, and this responsiveness can be inhibited if the initial focus is too rigid. Dray et al [3] reported that flexibility of focus was one of the key requirements for their methodology, and one of their regular weekly tasks was to refine the study focus.

\subsubsection{More time/bigger budget}

Nine respondents mentioned issues relating to timescales and budgets. Time pressures were described as being mainly due to fitting in with development timescales and analyzing large data sets; these restrictions mean that a larger budget would be required if more data was to be collected and analysed in the short time available between design and development cycles. Three more respondents commented that the usefulness of the methods was relative to the constraints under which they are used, particularly time constraints.

\section{DISCUSSION}

In general all groups of respondents tended to agree on the main advantages and disadvantages of lab and field studies, and according to their relative pros and cons they will be useful in different situations. However there is a need for considerable improvement in both field methods and in the resources available to inform their implementation. We discovered a definite lack of literature and other resources to inform implementation of field studies, and numerous different types of improvements were suggested by respondents. The survey found that utilization of results is less than optimal, and quality of results was frequently affected by cost constraints and deadlines. This suggests a need for improvements in field 
methods in order to address these issues and implement some of the improvements suggested by respondents.

We suggest that two of the main areas of potential improvement for field methods are improvements in the tools available for data collection and analysis, and improvements in methodologies (particularly in terms of their adaptability), since these opportunities were suggested by far the largest number of respondents. Improvements to tools are perhaps the most straightforward option with the clearest appreciable benefits, whereas creating more adaptable methods is likely to require more in-depth research and experimentation. Standardization of methodologies would be a good first step in this direction, since at present (according to the survey results) more practitioners appear to be using individual techniques such as observations and interviews rather than defined methods such as Contextual Design, and they also appear to perceive these techniques to be more effective.

Much of the skill of being able to adapt a method lies in being able to fully understand that method and appreciate its scope, so perhaps the provision of more guidelines and examples would enable practitioners to better educate themselves regarding the options available to them. For this reason we recommend increased publication of real-world examples for practitioners to follow, and also of "cookbooks" describing step-by-step how particular methods can be applied and adapted. While we do not deny that there is a need for training in research methods (as suggested in [8]) we still believe that this does not preclude the possibility of a recipe-style approach for practitioners who are unable to access training. We do not dispute the fact that methodological knowledge is required in order to be able to conduct field studies; the problem is that this methodological knowledge is not freely available in a written format for the benefit of practitioners who are unable to attend training courses. In real-life field studies are and will continue to be implemented by people who are doing it for the first time, and who do not have a good theoretical understanding of the methods and their application. This "cookbook" style approach has already been taken by some practitioners; for example the Rapid Contextual Design methodology presented by Holtzblatt et al [9]. Making methods more accessible to practitioners in this way is only likely to improve usage of field methods.

At present (according to our survey) only a small number of practitioners (approximately 20\%) are focused exclusively on field studies, the results of field studies are sometimes pressured by cost constraints and deadlines to the extent where the quality of results are affected, and in a high proportion of cases the results are not efficiently utilized. Field studies appear to be used predominantly by commercial practitioners, and they have relatively fewer proponents in academia than they do in industry. Clearly significant improvements to this situation are necessary if field study methodologies are to be further explored and improved by researchers and practitioners.

In general, field methods were used less for evaluation/validation than for any other purpose. Judging by the valuable results obtained by other practitioners when using field methods for evaluation (e.g. [10]) it appears that a significant opportunity for field method use is being overlooked. The key areas in which field evaluation methods require development are focus, problem extraction and impact ratings [10], and further research and guidelines in these areas would be of benefit.

In relation to the use of field methods for design, the key issue to be addressed is the leap from the data (e.g. the affinity diagram) to the vision and design recommendations. Cockton [1] explains that "The implications of contextual data are only relevant to specific designs...given alternative design options, the data generally has alternative implications for each option as regards its fit to an expected context of use". There is also a need for improved design representations and for better methods of grounding design ideas in actual data. Most of all, the concrete design ideas themselves do not emerge from the data, but address it and interact with it in different ways. More research is needed on the use of concurrent engineering approaches (advancing design alongside field studies, and their synergies).

\section{CONCLUSION}

This paper presents a line of investigation that has been initiated with a survey; there is still much work to be done in this line of enquiry, but we feel we have been able to provide an overview of the current state of field study usage and some directions in which further work could proceed. This data represents a picture of the current state of field study usage in January 2007, and much of its value derives from the current nature of the data. We believe that it would have been valuable to complement this survey with in-depth interviews and studies; however we felt that delaying the publication of the survey material while waiting for an opportunity to conduct such interviews would detract from its current value. Future work could perhaps involve conducting such interviews, but this would be challenging considering that the data was gathered from international respondents who mostly wished to remain anonymous.

In a paper of this brevity it is only possible to give a high level overview of the results of the survey. However this paper conveys an understanding of our aims in implementing this survey and also of the trends of the results that we uncovered. Whilst much of what we have discovered is already taken to be common knowledge by many practitioners, it has never previously been formally confirmed by a survey such as this, and the survey is therefore a valuable contribution to current HCI knowledge.

On the basis of these results we believe that there is still a significant amount of work to be done in optimizing field study methodologies. The field method improvements suggested by our respondents would be a good starting point for anyone wishing to further explore the development and adaptation of field study methodologies. In particular, we recommend that future research should focus on improvements in tools for data collection and analysis, and on improvements in adaptability of methodologies, as our survey suggested that these areas are of importance to a large proportion of practitioners. We also recommend the development of a toolkit of methods and tools that practitioners could mix and match to suit specific situations; this could prove to be extremely valuable and would address many of the concerns raised in this survey. Systematization and adaptation of field methods are also important aims for future research, as this will go some way towards addressing the time and budget concerns surrounding field studies, and will also increase accessibility of methods for practitioners, as will the increased publication of real-world examples for practitioners to follow.

\section{ACKNOWLEDGMENTS}

We wish to thank everyone who completed the online survey. Thanks also to Jaakko Lehikoinen (Nokia Research Center, Finland) and Ristomatti Partanen (RealityCheck Consulting) for their input on survey planning. 


\section{REFERENCES}

1. Cockton, G. (2002) "L'Avenir de L'Interface - The Future of the Interface". Actes des 2èmes journées Multimédia, ed. M. Noirhomme-Fraiture, Presses Universitaires de Namur, Belgium. ISBN 2-87037-375-9, pp. 11-34

2. Cockton, G., Woolrych, A., \& Hindmarch, M. (2004) "Reconditioned Merchandise: extended structured report formats in usability inspection". Proc. CHI 2004, 1433-1436

3. Dray, S., Siegel, D., Feldman, E. \& Potenza, M.(2002) "Why do version 1 and not release it? Conducting field trials of the tablet PC". Interactions March/April 2002

4. Dye, K. (2001) "As Easy to Use as a Banking Machine". Proc. HCI 2001

5. Forsythe, D. E. "It's Just a Matter of Common Sense": Ethnography as Invisible Work. Computer Supported Cooperative Work (CSCW), 8, 1 (1999), 127-145.).

6. Gulliksen, J., Boivie, I., Persoon, J., Hektor, A. \& Herulf, L. (2004) "Making a Difference: a survey of the usability profession in Sweden". NordiCHI 2004, 207-215

7. Gunther, R., Janis, J., \& Butler, S. (2001) “The UCD Decision Matrix: How, When and Where to Sell UserCentered Design into the Development Cycle". http://www.ovostudios.com/upa2001/ Accessed on September 17, 2007

8. Hughes J., King V., Rodden T., Andersen H. (1994), Moving Out from the Control Room: Ethnography in System Design, CSCW, October, 1994, USA, 429-439.

9. Holtzblatt, K., Wendell, J. \& Wood, S. (2005) "Rapid Contextual Design”. Morgan Kaufmann Publishers Inc, US

10. McDonald, S., Monahan, K. \& Cockton, G. (2006) "Rapid Contextual Design as a Field Evaluation Method". Proc. NordiCHI 2006, 437-440

11. Randall, D., Harper, R. \& Rouncefield, M. (2007) "Fieldwork for Design: Theory and Practice". Springer. ISBN: 978-1-84628-767-1

12. Rosenbaum, S., Rohn, J.A., \& Humburg, J. (2000) “A toolkit for strategic usability: Results from workshops, panels, and surveys". Proc. CHI 2000, 337-344

13. Vredenburg, K., Mao, J., Smith, P.W. \& Carey, T. (2002) "A survey of user-centered design practice". Proc. CHI 2002, CHI Letters 4(1), 471-478

14. Mao, J.Y., Vredenburg, K., Smith, P.W. \& Carey, T. (2005) "The State of User-Centered Design Practice". Communications of the ACM 10(3), March 2005

15. Venturi, G., Troost, J. and Jokela, T. (2006) "People, Organizations and Processes: an Enquiry into the Adoption of User Centered Design in the Industry". In International Journal of Human Computer Interaction, Vol 21, Issue 2. November 2006. 219-238

16. Wixon D.R., Ramey J. (eds.) (1996), Field Methods Casebook for Software Design. John Wiley \& Sons, Inc.

17. Wixon D.R., Ramey J., Holtzblatt K., Beyer H., Hackos J., Rosenbaum S., Page C., Laakso S. A., Laakso K.P. (2002), Usability in Practice: Field Methods Evolution and Revolution. In Proceedings of CHI 2002 Minneapolis MN, April, 2002, p.880-884. 